\title{
Anti-inflammatory activity of compounds isolated from Astragalus sinicus L. in cytokine-induced keratinocytes and skin
}

\author{
Byung-Hak Kim ${ }^{1}$, Ikhoon $\mathrm{Oh}^{2}$, Jung-Ho Kim ${ }^{1}$, Ju-eun Jeon ${ }^{2}$, Byeongwook Jeon ${ }^{1}$, Jongheon Shin ${ }^{2}$ \\ and Tae-Yoon Kim ${ }^{1}$
}

Inflammation is a part of the complex biological responses of a tissue to injury that protect the organ by removing injurious stimuli and initiating the healing process, and is considered as a mechanism of innate immunity. To identify biologically active compounds against pathogenic inflammatory and immune responses, we fractionated water, aqueous methanol and $n$-hexane layers from nine kinds of leguminosae and examined anti-inflammatory activity of the fractions in human keratinocytes and mouse skin. Among the fractions, rf3 and rf4, isolated from the aqueous methanol layer of Astragalus sinicus L., exhibited the strongest reactive oxygen species (ROS)-scavenging and anti-inflammatory activities as measured by inhibition of the intracellular ROS production, nuclear factor-kappaB (NF-кB), janus kinase (JAK)/signal transducer and activator of transcription (STAT), and phosphatidylinositol 3-kinase/Akt signaling in cytokine-stimulated human keratinocytes, as well as by effects on T-cell differentiation in mouse CD4 ${ }^{+} \mathrm{T}$ cells. In addition, topical application of rf3 and rf4 suppressed the progression of psoriasis-like dermatitis and expression of pro-inflammatory mediators in interleukin (IL)-23-injected mouse ears. Our results suggest that Astragalus sinicus L. may ameliorate chronic inflammatory skin diseases due to its antioxidant and antiinflammatory activities via regulation of the intracellular ROS production, NF-KB, JAK/STAT and PI3/Akt signaling cascades as well as immune responses, and these results are the first report that Astragalus sinicus L. exhibits pharmacological activity. Experimental \& Molecular Medicine (2014) 46, e87; doi:10.1038/emm.2013.157; published online 21 March 2014

Keywords: Astragalus sinicus L.; immune response; inflammation; JAK/STAT; NF-kB; PI3/Akt

\section{INTRODUCTION}

Although inflammation, induced by tissue injury and environmental stimuli, is a biological response designed to protect the organs from damage, prolonged exposure to such stimuli may result in chronic inflammation, which is considered as an inducer of inflammatory diseases and cancer. A number of signaling cascades are associated with the stages from initiation to maintenance of these inflammatory conditions. During inflammatory responses, numerous intracellular and extracellular signals, antigen receptors and pro-inflammatory cytokines activate janus kinase (JAK)/signal transducer and activator of transcription (STAT), ${ }^{1,2}$ phosphatidylinositol 3kinases $(\mathrm{PI} 3 \mathrm{~K}) / \mathrm{Akt}$, mitogen-activated protein kinases $^{3}$ and nuclear factor-kappaB $(\mathrm{NF}-\kappa \mathrm{B})^{4,5}$ signaling pathways. Although these signaling pathways are important to regulate physiological functions under normal condition, their aberrant activation is associated with a wide range of inflammatory and immune disorders, and cancer. ${ }^{5-8}$

Astragalus sinicus L., also known as the Chinese milkvetch, is an herbaceous and scandent perennial legume traditionally grown in the rice fields of eastern Asia, found in central to southern China, Japan and Korea, and it is commonly used as a green manure and forage for animal food. It is known to contain free amino acids in the flower petals and pollen; triterpene glycosides, such as soyasaponin I-IV and sapogenol, in the seeds; and (3R)-(-)-isomucronulatol, daidzin, 7-hydroxy-3'-4'-methylene-dioxypterocarpan, stigmast-5-ene$3-\beta, 7 \alpha$-diol trigonlline and canavanine in the stems. ${ }^{9,10}$ However, the biological activities of isolated compounds from Astragalus sinicus L., including these known compounds, have been little examined. In this study, we isolated biologically active ingredients rf3 and rf4 from

\footnotetext{
${ }^{1}$ Department of Dermatology, College of Medicine, The Catholic University of Korea, Seoul, Republic of Korea and ${ }^{2}$ College of Pharmacy, Seoul National University, Seoul, Republic of Korea

Correspondence: Professor T-Y Kim, Department of Dermatology, College of Medicine, The Catholic University of Korea, 505 Banpo-dong, Seocho-gu, Seoul 137-701, Republic of Korea.

E-mail: tykimder@catholic.ac.kr
}

Received 4 August 2013; revised 11 November 2013; accepted 15 November 2013 
the aqueous methanol fraction of Astragalus sinicus L. and examined their associated anti-inflammatory activities. Both rf3 and rf4 exhibited intracellular reactive oxygen species (ROS)-scavenging and anti-inflammatory activities, and they also suppressed $\mathrm{CD}^{+}$T-cell differentiation. Furthermore, rf3 and rf4 ameliorated progression of the inflammatory skin disease psoriasis. These activities were accompanied by inhibition of NF- $\mathrm{BB}$, JAK/STAT and PI3K/ Akt signaling.

\section{MATERIALS AND METHODS}

\section{Plant materials and extraction of active compounds}

Nine kinds of leguminosae plants were purchased from the Kyungdong-Mart, Seoul, Korea, in March 2009. The leguminosae plants used in this experiment were Cassia obtusifolia L., Albizia julibrissin Durazz., Astragalus membranaceus Bunge, Glycyrrhiza uralensis Fisch., Astragalus sinicus L., Gleditsia japonica Miq., Pueraria lobata Ohwi, Caragana sinica Rehder and Styphnolobium japonicum (L.) Schott, and a voucher specimen is deposited at the National Products Research Institute, College of Pharmacy, Seoul National University.

Extraction of the dried roots or stems from plants was described in Figure 1a. Briefly, the dried roots or stems were extracted repeatedly with methanol and dichloromethane. The crude extracts (CE) were partitioned into water $\left(\mathrm{H}_{2} \mathrm{O}\right)$ and $n$-butanol, and the latter fraction was repartitioned into $15 \%$ aqueous methanol $(\mathrm{MeOH})$ and $n$-hexane. On the basis of bioactivity tests, we isolated eight fractions from the aqueous $\mathrm{MeOH}$ fraction of Astragalus sinicus L.

\section{Cell line and reagents}

The immortalized human keratinocyte cell line HaCaT was purchased from CLS Cell Line Service GmbH (Eppelheim, Germany) and maintained in Dulbecco's modified Eagle's medium supplemented with $10 \%$ fetal bovine serum, $2 \mathrm{~mm}$ L-glutamine and antibiotics.

Recombinant mouse cytokines against interleukin (IL)-2, IL-4, IL-6 and IL-12, and mouse antibodies against anti-IL-4, anti-IFN- $\gamma$ and anti-CD28 were purchased from BD Biosciences (San Jose, CA, USA). Recombinant human TNF- $\alpha$, IFN- $\gamma$ and TGF- $\beta$ were obtained from PROSPEC (East Brunswick, NJ, USA) and recombinant mouse IL-23 was obtained from eBioscience (San Diego, CA, USA).

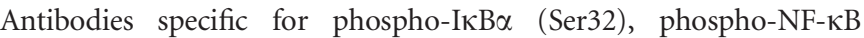
p65 (Ser536), phospho-STAT1 (Tyr701), STAT1, phospho-STAT3 (Tyr705), phospho-Akt (Ser473), Akt, phospho-ERK1/2 (Thr202/Tyr204), ERK1/2, phospho-p38 (Thr180/Tyr182), p38, phospho-JNK (Thr183/Tyr185), JNK, phospho-Src (Tyr416), Src, phospho-Lck (Tyr505), Lck, phospho-Lyn (Tyr507), Lyn, cyclooxygenase-2 (COX-2), intercellular adhesion molecule 1 (ICAM-1), PARP, cleaved caspase-3 (Asp175), cleaved caspase-9 (Asp330), p21, p27, Bax and GAPDH were purchased from Cell Signaling Technology (Danvers, MA, USA), and antibodies specific for I $\kappa \mathrm{B} \alpha, \mathrm{NF}-\kappa \mathrm{B}$ p65, STAT3 and Lamin B1 were purchased from Santa Cruz a

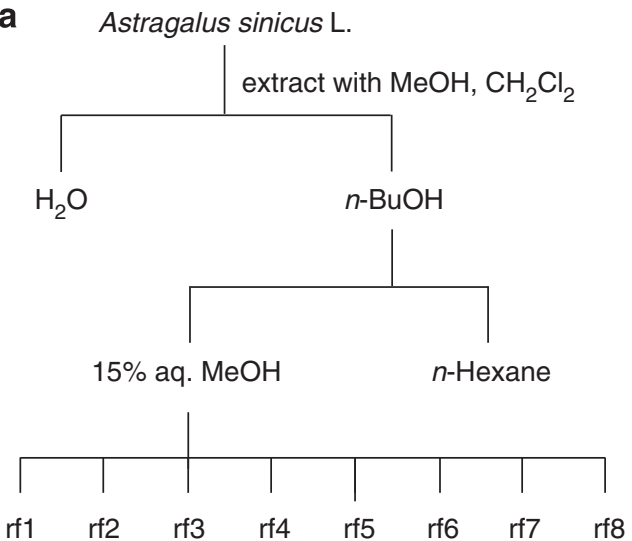

b
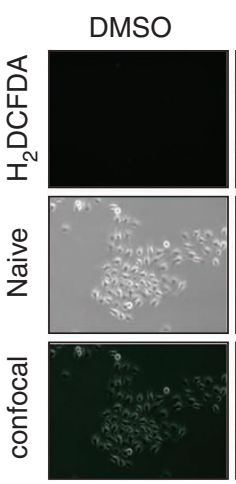

UVB

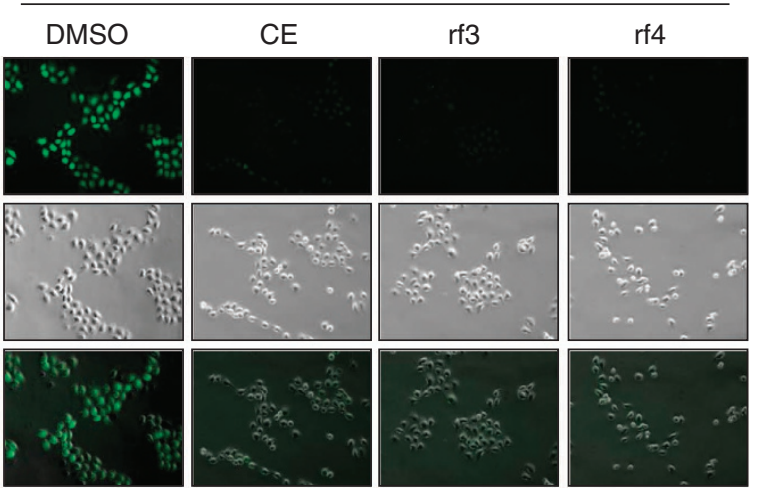

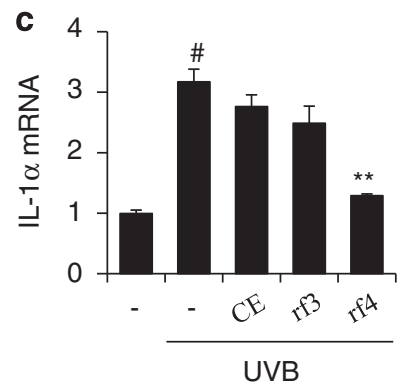
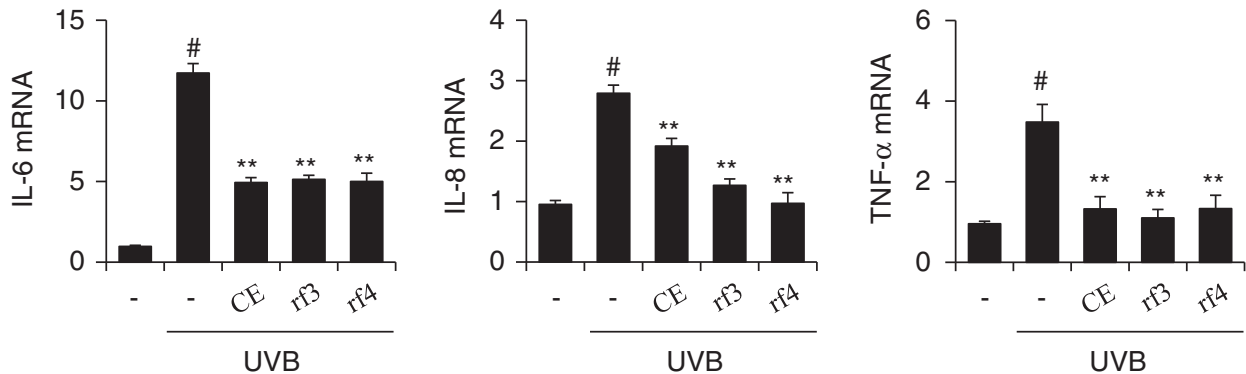

Figure 1 Active compounds isolated from Astragalus sinicus L. have antioxidant activity and inhibit the expression of pro-inflammatory mediators. (a) Isolation scheme of active compounds rf3 and rf4 from the aqueous methanol fraction of Astragalus sinicus L. (b) Fractions rf3 and rf4 exhibit ROS-scavenging activity in UVB-simulated HaCaT cells. (c) The mRNA levels of pro-inflammatory mediators were determined by quantitative real-time PCR in UVB-stimulated HaCaT cells. Results were normalized to the GAPDH signal and presented as -fold change relative to the vehicle-treated group. Results are expressed as the mean \pm s.e.m. of three independent experiments. ${ }^{\#} P<0.001$ versus vehicle-treated group; ${ }^{* *} P<0.001$ versus UVB-stimulated group. CE, crude extracts; DMSO, dimethyl sulfoxide; ROS, reactive oxygen species; UVB, ultraviolet B. 
Biotechnology (Santa Cruz, CA, USA). Horseradish peroxidaseconjugated goat anti-rabbit and anti-mouse antibodies were obtained from Life Technologies (Grand Island, NY, USA). All other chemicals were purchased from Sigma-Aldrich (St. Louis, MO, USA), unless otherwise noted.

\section{SDS-polyacrylamide gel electrophoresis and western blot analysis}

Cell pellets were lysed for $30 \mathrm{~min}$ at $4{ }^{\circ} \mathrm{C}$ in a lysis buffer containing $50 \mathrm{~mm}$ Tris- $\mathrm{HCl}$ (pH 7.4), $350 \mathrm{~mm} \mathrm{NaCl,} 1 \%$ Triton X-100, $0.5 \%$ Nonidet P-40, $10 \%$ glycerol, $0.1 \%$ SDS, 1 mm EDTA, 1 mm EGTA, $1 \mathrm{~mm} \mathrm{Na} \mathrm{VO}_{4}, 1 \mathrm{~mm}$ phenylmethylsulphonyl fluoride and phosphatase inhibitor cocktails. Whole-cell extracts were resolved on SDSpolyacrylamide gel electrophoresis and transferred onto polyvinylidene difluoride membranes (Pall Corporation, Pensacola, FL, USA). The membranes were blocked in a blocking buffer and incubated with specific primary antibodies for the target molecules at $4{ }^{\circ} \mathrm{C}$ overnight. The signals were detected using an ECL detection kit (iNtRON Biotechnology, Daejeon, Korea), followed by incubation with horseradish peroxidase-conjugated secondary antibodies.

\section{RNA isolation and quantitative real-time PCR}

Total RNA was isolated from cells or tissues using an RNeasy Mini Kit (Qiagen, Valencia, CA, USA) and complementary DNA was synthesized using a QuantiTect Reverse Transcription Kit (Qiagen). Quantitative real-time PCR was performed using the KAPA SYBR fast qPCR Kit (KAPA biosystems, Woburn, MA, USA) as previously described ${ }^{11}$ and the results were normalized to glyceraldehyde 3-phosphate dehydrogenase (GAPDH) gene expression. The PCR conditions were 1 cycle at $95^{\circ} \mathrm{C}$ for $5 \mathrm{~min}$, followed by 35 cycles at $96^{\circ} \mathrm{C}$ for $20 \mathrm{~s}, 60^{\circ} \mathrm{C}$ for $20 \mathrm{~s}$ and $72{ }^{\circ} \mathrm{C}$ for $20 \mathrm{~s}$, and ending with one cycle at $72{ }^{\circ} \mathrm{C}$ for $5 \mathrm{~min}$. Primers used in this experiment were purchased from Qiagen.

Isolation and in vitro differentiation of mouse $\mathrm{CD} 4^{+} \mathrm{T}$ cells Naive $\mathrm{CD} 4^{+} \mathrm{T}$ cells were purified and differentiated from spleens and lymph nodes of C57BL/6 mice as previously described. ${ }^{12}$ Briefly, cells were enriched using mouse T-cell enrichment column (R\&D Systems, Minneapolis, MN, USA) and purified by negative selection using MACS column (Miltenyi Biotech Inc., Auburn, CA, USA). The cells were activated by plate-bound anti-CD3 antibody in 96-well plate (BD Biosciences) and soluble anti-CD28 antibody $\left(2 \mu \mathrm{g} \mathrm{ml}^{-1}\right)$ in RPMI 1640 medium supplemented with $10 \%$ fetal bovine serum, $2 \mathrm{~mm}$ glutamine and antibiotics for 4 days. The cells were polarized under Th1 polarizing condition $\left(10 \mathrm{ng} \mathrm{ml}^{-1} \mathrm{IL}-12\right.$ and $10 \mu \mathrm{g} \mathrm{ml}^{-1}$ anti-IL-4 antibody), Th2 polarizing condition $\left(20 \mathrm{ng} \mathrm{ml}^{-1} \mathrm{IL}-4\right.$ and $10 \mu \mathrm{g} \mathrm{ml}^{-1}$ anti-IFN- $\gamma$ antibody), Th17 polarizing condition (20 $\mathrm{ng} \mathrm{ml}^{-1}$ IL-6, $5 \mathrm{ng} \mathrm{ml}^{-1}$ TGF- $\beta, 10 \mu \mathrm{g} \mathrm{ml}^{-1}$ anti-IFN- $\gamma$ antibody and $10 \mu \mathrm{g} \mathrm{ml}^{-1}$ anti-IL-4 antibody) or Treg polarizing condition ( $5 \mathrm{ng} \mathrm{ml}^{-1}$ TGF- $\beta$ and $10 \mathrm{ng} \mathrm{ml}^{-1}$ IL-2).

\section{Measurement of cytokine and prostaglandin production}

Cytokine and prostaglandin $\mathrm{E}_{2}$ amounts were measured by ELISA (R\&D Systems) using cultured supernatant according to the manufacturer's protocol. The supernatant was collected from mouse CD4 ${ }^{+}$ $\mathrm{T}$ cells after differentiation for 4 days or HaCaT cells after stimulated for $24 \mathrm{~h}$ by combination with TNF- $\alpha$ and IFN- $\gamma$ in the presence or absence of the compound.

\section{Transfection and NF- $\kappa \mathrm{B}-$ dependent reporter assay}

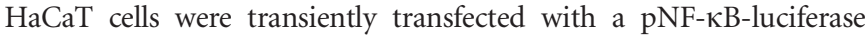
construct and pRL-TK control vector using a Lipofectamine 2000 (Life Technologies). Twenty four hours after transfection, the cells were stimulated with TNF- $\alpha$ and IFN- $\gamma$ in the presence or absence of

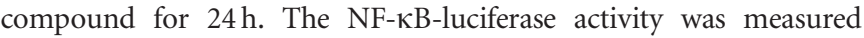
using a Dual-Luciferase Assay Kit (Promega, Madison, WI, USA) according to the manufacturer's protocol, and the firefly luciferase activities were normalized to the Renilla luciferase activity.

\section{Measurement of the intracellular ROS production}

$\mathrm{HaCaT}$ cells were seeded at $3 \times 10^{5}$ cells/well in six-well plates. After overnight, cells were starved in serum-free Dulbecco's modified Eagle's medium media and stimulated with ultraviolet B (UVB, $100 \mathrm{~mJ} \mathrm{~m}^{-2}$ ) or by combination with TNF- $\alpha$ and IFN- $\gamma$ for $4 \mathrm{~h}$, followed by pre-treatment with compound for $1 \mathrm{~h}$. The cells were stabilized in Hanks' Balanced Salt Solution for 30 min, stained with $2^{\prime}, 7^{\prime}$-dichlorofluorescein diacetate $\left(\mathrm{H}_{2} \mathrm{DCFDA}, 10 \mu \mathrm{m}\right)$ in a $37^{\circ} \mathrm{C}$ incubator for $30 \mathrm{~min}$ and then immediately analyzed for intracellular ROS-scavenging activity using a confocal microscopy (Carl Zeiss, Jena, Germany).

\section{Immunofluorescence analysis}

Serum-starved $\mathrm{HaCaT}$ cells $\left(5 \times 10^{5}\right.$ cells/well $)$ were pre-treated with either vehicle or compound for $1 \mathrm{~h}$ and then stimulated with TNF- $\alpha$ and IFN- $\gamma$ for $1 \mathrm{~h}$. Immunostained NF- $\mathrm{KB}$ p 65 signal was detected as described previously. ${ }^{13}$ Briefly, cells were fixed in $4 \%$ paraformaldehyde, permeabilized in $0.5 \%$ Triton $\mathrm{X}-100$ and then blocked in phosphate-buffered saline containing $1 \%$ bovine serum albumin. For immunostaining, the cells were incubated with anti-NFКB p65 antibody for $2 \mathrm{~h}$, washed three times in blocking buffer and incubated with Alexa Fluor 568 anti-rabbit IgG antibody (Molecular Probe, Eugene, OR, USA) for $1 \mathrm{~h}$. Immunostained NF-kB p65 was analyzed using a confocal fluorescence microscope (Carl Zeiss, Jena, Germany) and nuclei were counterstained with Hoechst (Molecular Probe).

\section{IL-23-induced psoriasis-like cutaneous inflammatory model} All procedures of animal experiments were approved by the Catholic Research Institute of the Medical Science Committee on Institutional Animal Care and Use, and performed in accordance with the institution guidelines. C57BL/6 mice were acclimatized for 1 week in specific pathogen-free conditions and maintained in a room at constant temperature $\left(23 \pm 2^{\circ} \mathrm{C}\right)$ on a 12 -h light/dark cycle with free access to laboratory chow diet and water. Vehicle (phosphate-buffered saline) or recombinant mouse IL-23 (500 ng/10 $\mu \mathrm{l})$ was intradermally injected into mice ears $(n=5)$, followed by topical application of compound every other day for 14 days as previously described. ${ }^{12-14}$ Ear thickness was measured $24 \mathrm{~h}$ after final injection, and the ears were collected and stored at $-80^{\circ} \mathrm{C}$ for further experiments.

\section{Histological analysis}

Mouse ears were fixed with $4 \%$ paraformaldehyde in phosphatebuffered saline for $24 \mathrm{~h}$, washed with tap-water, dehydrated with grade ethanol and then embedded in paraffin. The paraffin blocks were cut in $4-\mu \mathrm{m}$ thick sections, mounted on glass slides, dewaxed, rehydrated with grade ethanol and then stained with hematoxylin-eosin (H\&E). Analysis was carried out using a fluorescence attached microscope (Olympus, Tokyo, Japan). 


\section{Statistical analysis}

Data were expressed as mean \pm s.e.m. Two-tailed Student's $t$-test was used to show a statistical significance in case of multiple comparisons to consider $P$ values at a level of $<0.001$ or 0.05 .

\section{RESULTS}

\section{Astragalus sinicus L. extracts exhibit biological activity}

In all studies, unless otherwise noted, $\mathrm{CE}$ of Astragalus sinicus $\mathrm{L}$., and rf3 and rf4 isolated from the CE were tested at a concentration of $20 \mu \mathrm{g} \mathrm{ml}^{-1}$ and $10 \mu \mathrm{g} \mathrm{ml}^{-1}$, respectively. Samples were pre-treated for $1 \mathrm{~h}$ before stimulation by a combination of TNF- $\alpha\left(10 \mathrm{ng} \mathrm{ml}^{-1}\right)$ and IFN- $\gamma\left(100 \mathrm{U} \mathrm{ml}^{-1}\right)$.

To identify biologically active compounds that inhibit inflammatory responses, we fractionated polar and non-polar layers from nine kinds of leguminosae plants. From the fractionates, we identified that the aqueous methanol layer of Astragalus sinicus L. exhibited the strongest biological activity as measured by inflammatory response in either cytokine- or UVB-induced human keratinocytes (data not shown). We, therefore, further purified eight fractions from the aqueous methanol layer of this plant (Figure 1a).

To test the biological activity of the eight fractions purified from the aqueous methanol layer of Astragalus sinicus L., we first examined their antioxidant activity. Although ROS production was not detected in vehicle-treated quiescent keratinocytes, the production was markedly increased by stimulation with either UVB or cytokines. Among the eight fractions, rf3 and rf4 exhibited the strongest ROS-scavenging activity in UVB- or cytokine-induced keratinocytes (Figure $1 \mathrm{~b}$ and Supplementary Figure S1).

\section{Astragalus sinicus L. extracts inhibit the expression and production of pro-inflammatory mediators}

We next determined the anti-inflammatory activity by measuring the levels of inflammatory mediators in UVB-stimulated human keratinocytes by quantitative real-time PCR analysis. Similar to their antioxidant activity, rf3 and rf4 effectively inhibited the mRNA levels of several pro-inflammatory mediators such as IL- $1 \alpha$, IL- 6 , IL- 8 and TNF- $\alpha$ (Figure $1 \mathrm{c}$ and Supplementary Figure S2). We, therefore, selected rf3 and rf4 as potential candidates possessing biological activities, including antioxidant and anti-inflammatory activities, for further experiments.

Similar results were observed in cytokine-stimulated human keratinocytes. The rf3 and rf4 effectively suppressed both protein and mRNA levels of COX-2 and ICAM-1, and the inhibitory activities of rf4 were similar to, or much stronger than, those of CE and rf3 (Figures $2 \mathrm{a}$ and $\mathrm{b}$ ). Together with the inhibition of COX-2 and ICAM-1 expression, rf3 and/or rf4 also effectively suppressed the mRNA levels of other proinflammatory mediators, such as IL- $1 \alpha$, IL- $1 \beta$, IL- 6 and TNF$\alpha$, in cytokine-stimulated human keratinocytes, and rf4 exhibited much stronger inhibitory activities than those of $\mathrm{CE}$ and rf3 (Figure 2c and Supplementary Figure S3a). In addition, the rf3 and rf4 effectively inhibited the production of prostaglandin $\mathrm{E}_{2}$ and IL-6 (Figure 2d). In contrast, the expression and production of anti-inflammatory cytokine IL10 were significantly increased by treatment with rf3 and rf4 in vitro (Figures $2 \mathrm{c}$ and $\mathrm{d}$ ). These results suggest that Astragalus sinicus L. extracts may be useful candidates in regulating inflammatory disorders.

\section{Astragalus sinicus $\mathrm{L}$. extracts suppress $\mathrm{CD}^{+}{ }^{+} \mathrm{T}$-cell differentiation}

To delineate whether Astragalus sinicus L. extracts can regulate certain immune responses, particularly with regard to $\mathrm{CD} 4^{+}$ T-cell differentiation, we examined the expression and production of T-cell-specific master regulatory factors and their signature cytokines in cultured mouse $\mathrm{CD} 4{ }^{+} \mathrm{T}$ cells under the appropriate polarizing conditions. The mRNA levels of Th2- and Th17-cell master regulatory factors, GATA-3 and ROR $\gamma$ t, respectively, were effectively suppressed by treatment with rf3 and rf4, whereas mRNA levels of the Th1-cell master regulatory factor T-bet were not affected. In contrast, treatment with rf3 and rf4 significantly increased mRNA levels of the Treg-cell master regulatory factor Foxp3 (Figure 3a).

We next examined the effects of Astragalus sinicus L. fractions on the expression of T-cell-specific signature cytokines of $\mathrm{CD} 4^{+}$T-cell subsets. ${ }^{15-17}$ The mRNA levels of Th2 and Th17 cytokines, such as IL-4, IL-17A and IL-22, were effectively decreased by treatment with $\mathrm{rf3}$ and $\mathrm{rf} 4$, whereas the mRNA levels of the Th1 cytokine TNF- $\alpha$, but not IFN- $\gamma$, was marginally decreased by treatment with rf3 only. In contrast, the mRNA levels of the anti-inflammatory cytokine IL-10, the Th2- and Treg-cell-signature cytokine, were significantly increased by treatment with rf3, but not rf4 (Figure 3b).

Consistent with the results of mRNA levels on T-cell-specific master regulatory factors and their signature cytokines, the production of Th17-cell-signature cytokine IL-17A was effectively inhibited by treatment with $\mathrm{rf3}$ and rf4 under Th17 polarization condition. In addition, the production of IL-10 in Th2 and Treg cells was significantly increased by treatment with rf3 and rf4 (Figure 3c). These results suggest that Astragalus sinicus L. extracts may be able to regulate inflammatory diseases, such as allergy, asthma and systemic autoimmune diseases, induced by excessive activated Th2 and Th17 cells.

\section{Astragalus sinicus L. extracts inhibit JAK/STAT and PI3K/ Akt signaling}

To determine the molecular mechanisms of the rf3 and rf4 on anti-inflammatory activity and on the inhibition of $\mathrm{CD} 4^{+}$ T-cell differentiation, we first examined whether rf3 and rf4 can regulate JAK/STAT signaling in cytokine-induced human keratinocytes. Cytokine-induced tyrosine phosphorylation of STAT1 and STAT3 was strongly inhibited by treatment with rf4, whereas CE and rf3 exhibited inhibitory activity only on STAT3, but weak to no inhibitory activity against STAT1 (Figure $4 \mathrm{a}$, lanes 1 and 3 ). Compared with the levels of tyrosine phosphorylation, total levels of these proteins were not altered (Figure $4 \mathrm{a}$, lanes 2 and 4 ). 

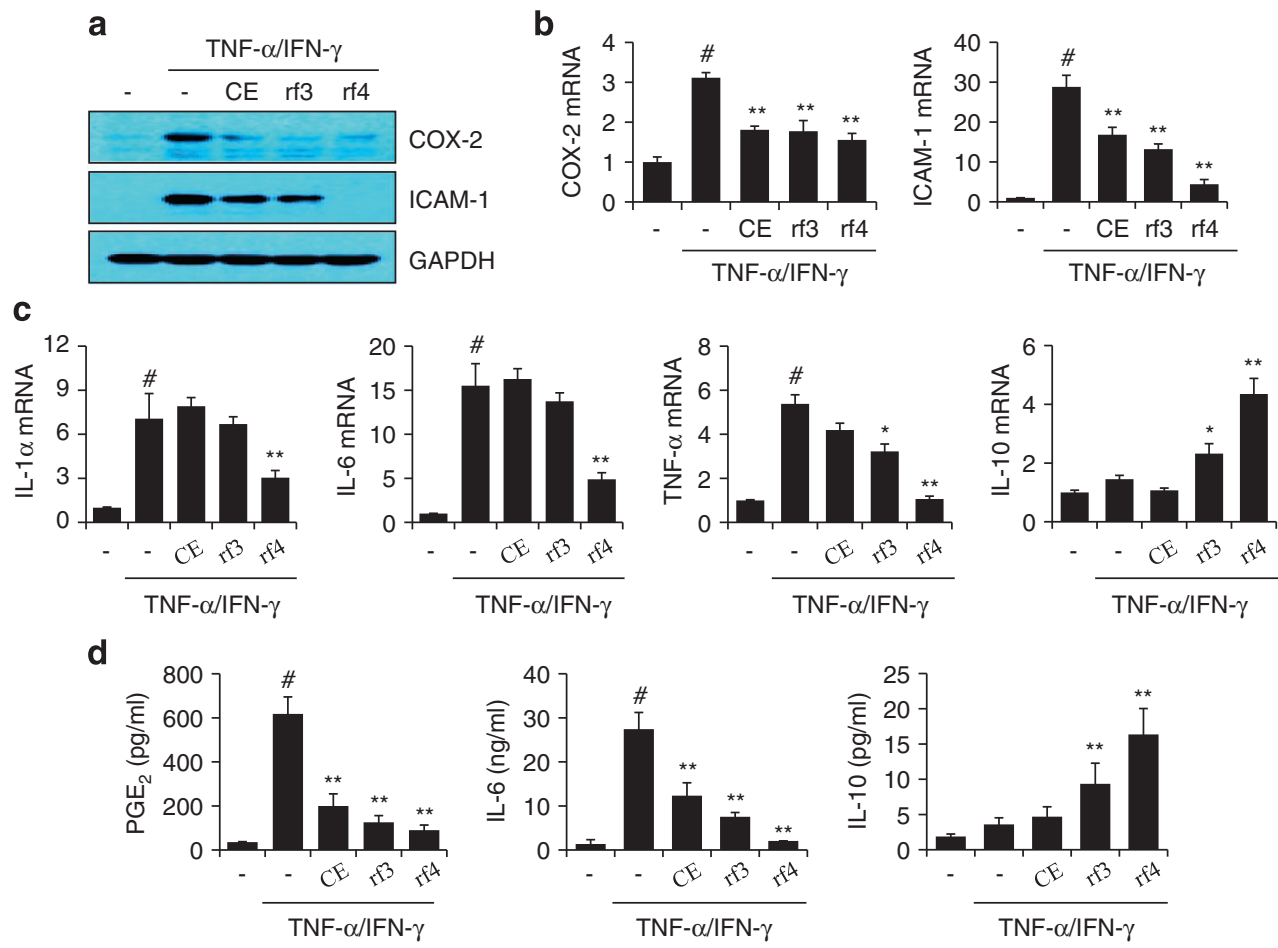

Figure 2 Inhibition of the expression of pro-inflammatory mediators by rf3 and rf4 in HaCaT cells. (a) Cells were stimulated with TNF- $\alpha$ and IFN- $\gamma$ for $24 \mathrm{~h}$ and western blot analysis was performed using anti-COX-2 and anti-ICAM-1 antibodies. Data are representative of three independent experiments with similar results, and GAPDH served as a loading control. (b-d) The mRNA levels of pro-inflammatory mediators were determined by qRT-PCR in HaCaT cells stimulated for $4 \mathrm{~h}$ with TNF- $\alpha$ and IFN- $\gamma$. Data were normalized to GAPDH signal and are presented as -fold change relative to the vehicle-treated group. Results are expressed as the mean \pm s.e.m. of three independent experiments. ${ }^{\#} P<0.001$ versus vehicle-treated group; ${ }^{*} P<0.001$ and ${ }^{*} P<0.05$ versus TNF- $\alpha$ - and IFN- $\gamma$-stimulated groups, respectively. CE, crude extracts; IL, interleukin; $\mathrm{PGE}_{2}$, prostaglandin $\mathrm{E}_{2}$; qRT-PCR, quantitative real-time PCR.

Among the non-JAK/STAT signaling pathways examined, rf3 and rf4 effectively inhibited only the PI3K/Akt signaling pathway, by inhibition of the phosphorylation of serine residue 473 of Akt (Figure 4a, lane 5). With respect to the IFN- $\gamma$-JAKdependent Src family and mitogen-activated protein kinase signaling pathways, rf3 and rf4 showed weak to no inhibitory effects, respectively, on these signaling, including Lyn, Src, Lck, ERK1/2, p38 and JNK, in cytokine-stimulated human keratinocytes (Figures $4 \mathrm{~b}$ and $\mathrm{c}$ ). These results indicate that $\mathrm{rf3}$ and rf4 inhibit JAK/STAT and non-JAK/STAT-dependent PI3K/Akt pathways in cytokine-stimulated human keratinocytes, and the inhibitory effects of rf4 are much stronger than those of CE or rf3.

\section{Astragalus sinicus L. extracts inhibit NF- $\mathrm{\kappa B}$ signaling cascades}

We next examined whether $\mathrm{rf3}$ and $\mathrm{rf} 4$ can regulate NF- $\kappa \mathrm{B}$ signaling in cytokine-stimulated human keratinocytes, because this signaling is known to be one of the important signals on inflammatory responses. ${ }^{7,18}$ In experiments on the phosphorylation states of inhibitory protein I $\mathrm{I} B \alpha$ and NF$\kappa \mathrm{B}$ p65 proteins, phosphorylation of both proteins was strongly inhibited by incubation with $\mathrm{rf} 4$, whereas $\mathrm{CE}$ and rf3 exhibited similar to no inhibitory effect than rf4 (Figure 5a, lanes 1 and 3). Degradation of IкB $\alpha$ protein was consistent with phosphorylation states of $I \kappa B \alpha$ proteins (Figure $5 \mathrm{a}$, lane 2 ), suggesting that the inhibitory targets of rf3 and rf4 are somewhat different.

To identify the localization of NF- $\kappa B$ p65, one of the NF- $\kappa B$ subunit proteins, we conducted western blot and immunofluorescence analysis. NF- $\mathrm{KB}$ p65 localized in the cytosolic region of quiescent keratinocytes, but almost all the protein is translocated to the nuclear region following cytokine stimulation. Nuclear translocation of NF- $\mathrm{\kappa B}$ p 65 was effectively inhibited by rf4, and this inhibitory effect was similar or much stronger than that of $\mathrm{CE}$ or rf3 (Figures $5 \mathrm{~b}$ and c). Further, we examined NF- $\mathrm{KB}$-dependent promoter activity and found that rf4 exhibited a little stronger inhibitory effect than that was seen with either rf3 or CE in cytokine-stimulated keratinocytes containing an NF- $\kappa B-l u c i f e r a s e$ system construct (Figure 5d). Therefore, rf3 and rf4, present in the aqueous methanol extracts of Astragalus sinicus L., possess anti-inflammatory activity through inhibition of the NF- $\kappa \mathrm{B}$ signaling pathway, and the inhibitory activity of $\mathrm{rf} 4$ is more potent than that of either CE or rf3 in cytokine-stimulated NF- $\mathrm{KB}$ signaling.

\section{Astragalus sinicus L. extracts suppress cutaneous} inflammatory damage

Because rf3 and rf4 inhibit the JAK/STAT, NF- $\mathrm{KB}$ and PI3K/ Akt signaling cascades, we examined whether these fractions 

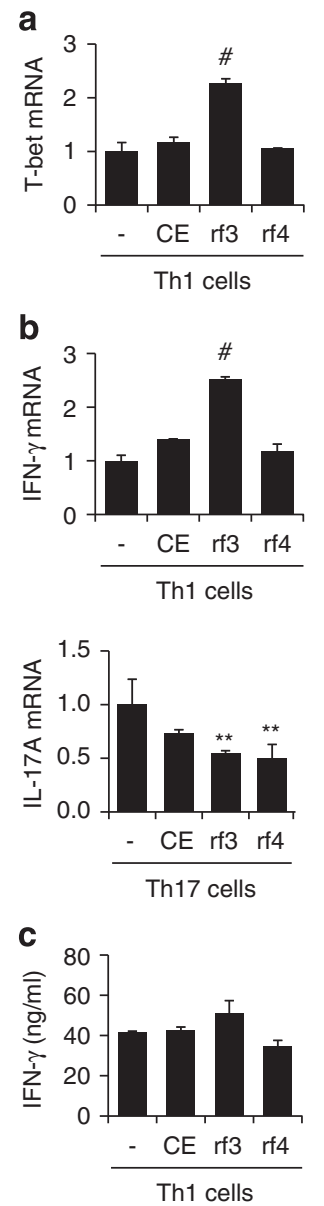
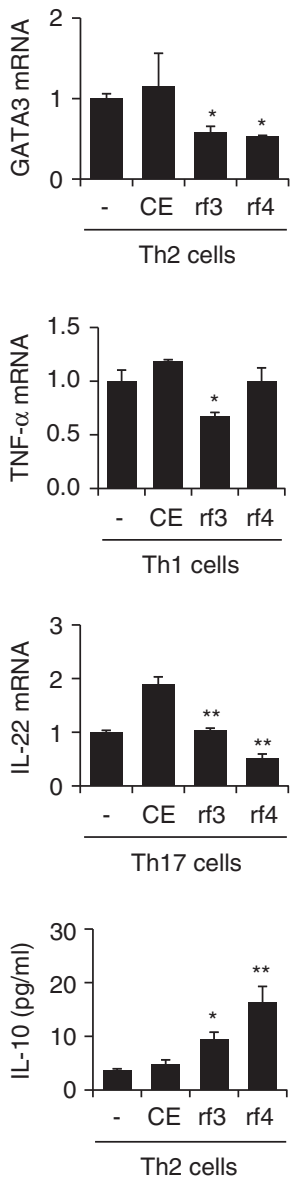
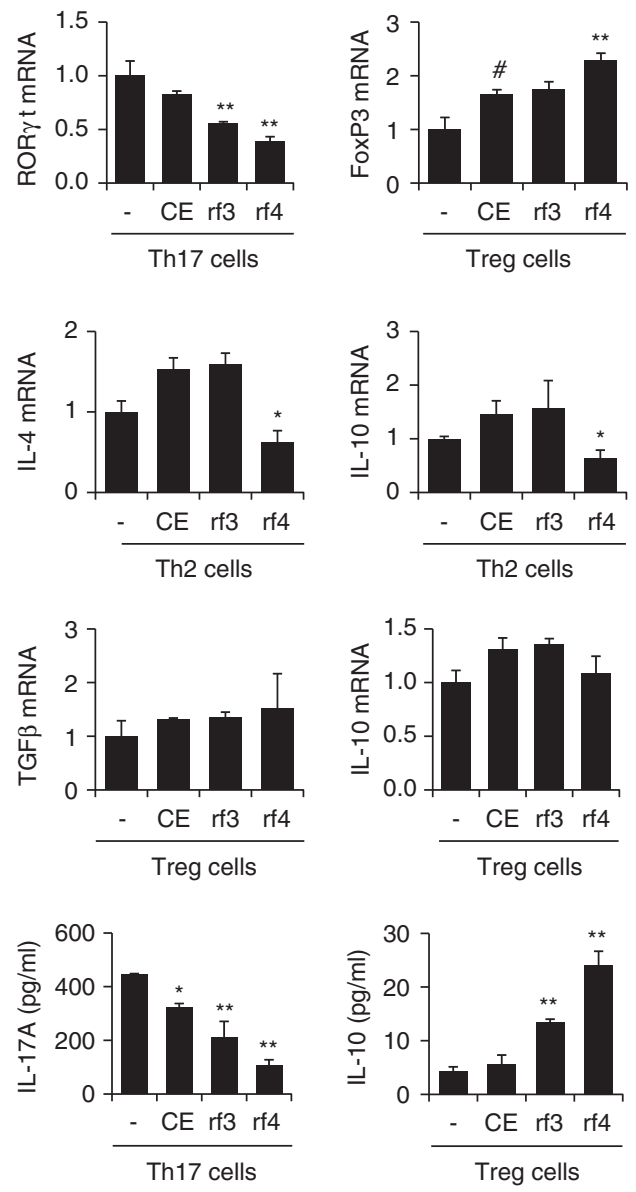

Figure 3 Inhibition of CD4 ${ }^{+}$T-cell differentiation by rf3 and rf4 in mouse CD4 ${ }^{+}$T cells. (a, b) Naive CD4 ${ }^{+}$T cells were cultured under the appropriate polarizing conditions for 4 days, as described in Materials and methods. Total RNA was isolated from the cells and quantitative real-time PCR was performed to analyze the expression of T-cell-lineage-specific master transcription factors (a) or cytokines (b). (c) Cytokine production was measured by ELISA in the supernatants of cultured T cells. ${ }^{\#} P<0.001$ versus not differentiated group; $* * P<0.001$ and ${ }^{*} P<0.05$ versus differentiated group. $\mathrm{CE}$, crude extracts; IL, interleukin.

a

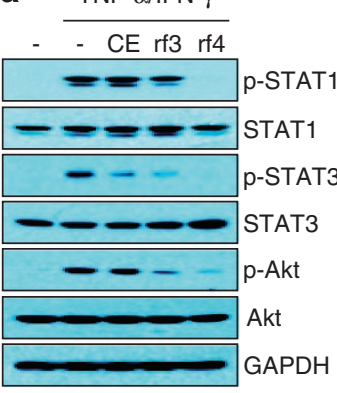

b TNF- $\alpha / \mathrm{IFN}-\gamma$

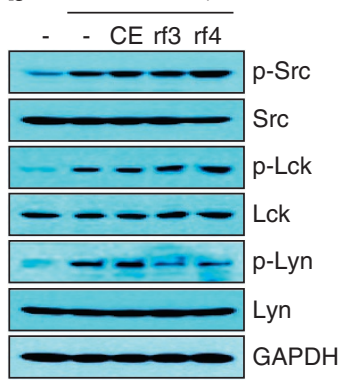

C TNF- $\alpha / \mathrm{IFN}-\gamma$

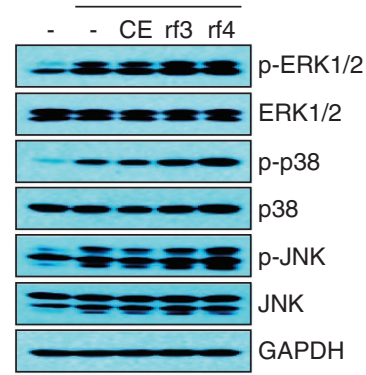

Figure 4 Inhibition of the JAK/STAT and PI3K/Akt signaling cascades by rf3 and rf4 in HaCaT cells. (a-c) Cells were stimulated with TNF- $\alpha$ and IFN- $\gamma$ for 15 min, followed by pre-treatment with rf3 or rf4 for $1 \mathrm{~h}$, and western blot analysis was performed with antibodies specific for the molecules indicated. Data represent one of the three independent experiments, all of which showed similar results. Levels of GAPDH served as a loading control. CE, crude extracts.

can regulate inflammatory skin damage induced by intradermal injection of IL-23 into mouse ears. Intradermal injection of IL-23 into mouse skin has recently been shown to induce a psoriasis-like epidermal hyperplasia, and this inflammation is dependent on increasing IL-17A and/or CCR6 expression. 12-14,19,20 We performed histopathological examination of H\&E-stained ear sections and observed a markedly increased skin thickness and progression of inflammatory 

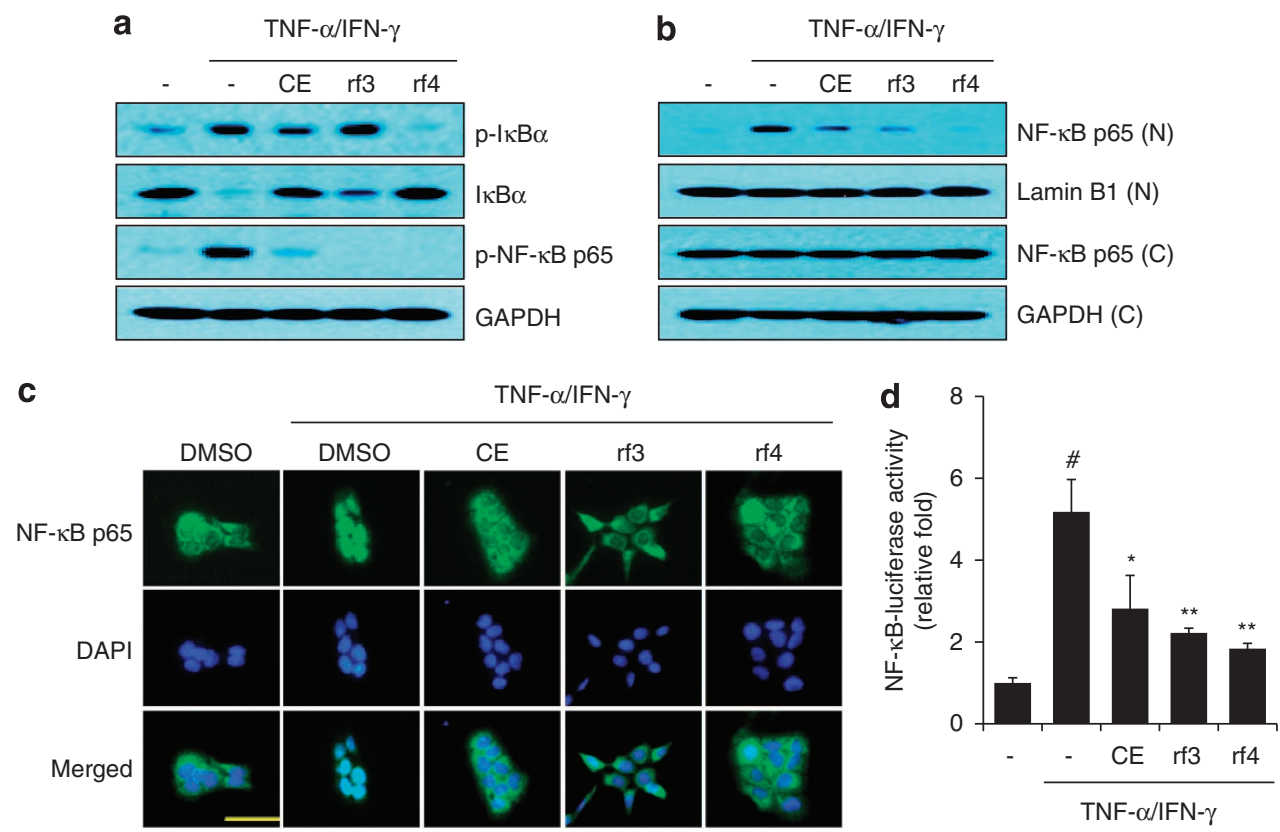

Figure 5 Inhibition of NF-kB signaling cascades by rf3 and rf4 in HaCaT cells. (a) Cells were stimulated with TNF- $\alpha$ and IFN- $\gamma$ for 15 or $30 \mathrm{~min}$, followed by pre-treatment with rf3 or rf4 for $1 \mathrm{~h}$, and western blot analysis was performed with antibodies for phospho-IKB $\alpha$, NF-

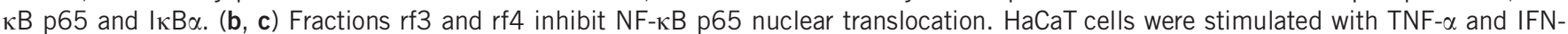
$\gamma$ for $1 \mathrm{~h}$, followed by pre-treatment with rf3 or rf4 for $1 \mathrm{~h}$, and nuclear translocation of NF-kB p65 was measured by western blot (b) and immunofluorescence analysis (c). $\mathrm{N}$ and $\mathrm{C}$ indicate nucleic and cytosolic protein, and the levels of GAPDH and Lamin B1 served as loading control for either cytosolic or nucleic, respectively. (d) Fractions rf3 and rf4 inhibit NF- $\mathrm{kB}$-dependent luciferase activity in TNF- $\alpha$ and IFN- $\gamma$-stimulated $\mathrm{HaCaT}$ cells. Results are expressed as the mean \pm s.e.m. of three independent experiments. ${ }^{\#} P<0.001$ versus vector-transfected group; ${ }^{* *} P<0.001$ and ${ }^{*} P<0.05$ versus $N F-\kappa B$-reporter-transfected group. CE, crude extracts; DMSO, dimethyl sulfoxide.

skin damage, such as epidermal hyperplasia (acanthosis), hyperkeratosis and dermal infiltration of inflammatory cells, in IL-23-injected mouse ears, as compared with vehicle injection. These pathological progressions were effectively decreased by topical application of rf3 and rf4 (Figure 6a and Table 1).

To examine the mRNA levels of pro-inflammatory mediators in the ears of the mice, we performed quantitative real-time PCR analysis. Consistent with the histological changes, the mRNA levels of known major inflammatory cytokines and chemokines in psoriasis, such as IL-17A, IL-22, CXCL1, CXCL10, CCL17, CCL20, CCL27 and CCR6, were markedly increased by IL-23 injection, as well as in cytokine-stimulated human keratinocytes, whereas the levels of these mRNAs were effectively decreased by rf3 and rf4 (Figure 6b and Supplementary Figure S3c). Together with these cytokines, the mRNA levels of various pro-inflammatory cytokines, including IL- $1 \alpha$, IL-1 $\beta$ and IL-6, were increased in IL-23-injected mouse ears, whereas these levels were also effectively decreased by rf3 and rf4 (Supplementary Figure S3b). In contrast, the mRNA levels of the anti-inflammatory cytokine IL-10 were significantly increased by rf3 or rf4 treatment (Figure 6b). These results suggest that Astragalus sinicus L. extracts might be able to suppress cutaneous inflammatory diseases such as psoriasis.

\section{DISCUSSION}

To identify biologically active ingredients against inflammatory disease, we fractionated water, aqueous methanol and $n$ hexane layers from nine kinds of leguminosae plants, and we examined their antioxidant and anti-inflammatory activities in human keratinocytes. Among the fractions, rf3 and rf4, isolated from the aqueous methanol layer of Astragalus sinicus L., exhibited the strongest antioxidant and anti-inflammatory activities.

With respect to the molecular mechanism of the antiinflammatory activity and immune regulation shown by rf3 and rf4, we demonstrated an effect on the JAK/STAT signaling cascade. Examination of rf3 and rf4 fractions on the JAK/STAT signaling pathway indicated that rf3 and rf4, and especially rf4, exhibit potential inhibitory activity in cytokine-induced human keratinocytes. By studying the inhibition of STAT activation in cytokine-stimulated human keratinocytes, we observed that rf3 and rf4 regulate differentiation of $\mathrm{CD} 4{ }^{+}$ $\mathrm{T}$ cells, specifically Th2 and Th17 cell differentiation were effectively inhibited; on the other hand, Treg-cell differentiation was enhanced. In parallel with these results, rf3 and rf4 strongly inhibited the expression of Th2- and Th17-lineagespecific cytokines, such as IL-4, IL-17A and IL-22, whereas the Treg cytokines TGF- $\beta$ and IL-10 were marginally increased, suggesting the therapeutic effects of rf3 and rf4 involved in the diseases of Th2 and Th17 cells, including allergy and asthma, 

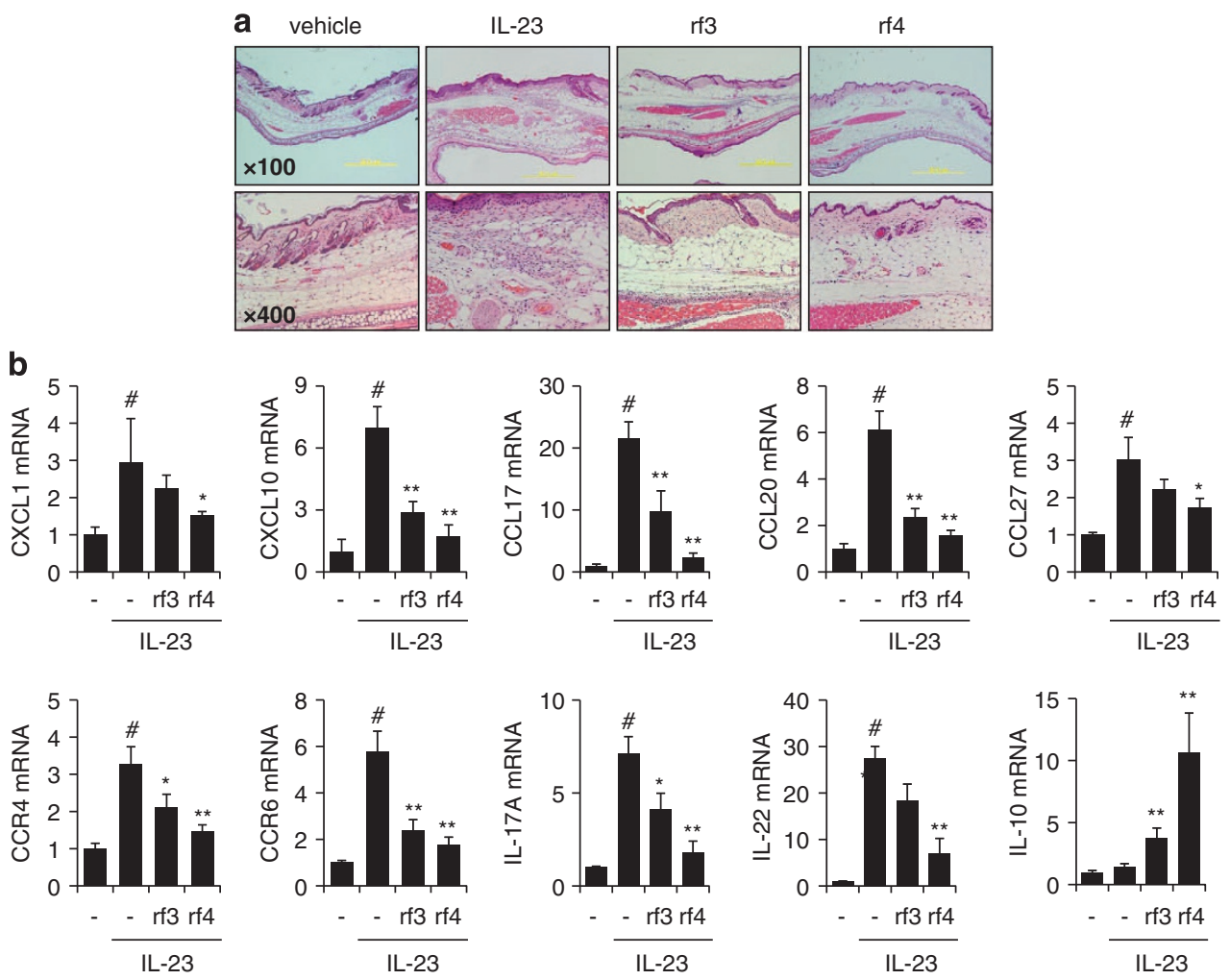

Figure 6 Amelioration of cytokine-induced cutaneous inflammation in mice skin by rf3 and rf4. (a) Mouse ears were topically treated with rf3 or rf4 and subsequently injected with phosphate-buffered saline or mouse IL-23 ( $500 \mathrm{ng} / 10 \mu \mathrm{l}$, each) into mouse ears every other day for 14 days ( $n=5$ mice per group). Histological assessment of the sections from the ears was performed by H\&E staining. (b) The mRNA levels of pro-inflammatory mediators were determined by quantitative real-time PCR in IL-23-injected mouse ears. Data were normalized to GAPDH signal and are presented as -fold change relative to the phosphate-buffered saline-treated group. Results are expressed as the mean \pm s.e.m. from three independent experiments. ${ }^{\#} P<0.001$ versus vehicle-treated group; ${ }^{* *} P<0.001$ and ${ }^{*} P<0.05$ versus IL-23treated groups. IL, interleukin.

Table 1 The ear thickness and inhibitory effect on ear swelling in IL-23-induced mouse ears

\begin{tabular}{lcc}
\hline Group & $\begin{array}{c}\text { Ear thickness }(\times 10 \mu \mathrm{m}, \\
\text { mean } \pm \text { s.e.m. })\end{array}$ & $\begin{array}{c}\text { Inhibition \% } \\
\text { (mean } \pm \text { s.e.m.) }\end{array}$ \\
\hline PBS & $41.3 \pm 1.8$ & - \\
IL-23 & $70.5 \pm 3.5$ & - \\
rf3 & $59.3 \pm 2.3$ & $38.5 \pm 5.1$ \\
rf4 & $50.0 \pm 2.0$ & $70.1 \pm 4.6$
\end{tabular}

Abbreviations: IL, interleukin; PBS, phosphate-buffered saline.

as well as systemic autoimmune diseases and cancer. Furthermore, among the activated signaling cascades, including JAKdependent and non-JAK/STAT signaling pathways, rf3 and rf4 effectively inhibited only PI3K/Akt signaling.

In addition to JAK/STAT signaling pathways, we observed that fractions rf3 and rf4, and especially rf4, result in strong inhibition of the NF- $\kappa \mathrm{B}$ activation by inhibition of the phosphorylation and degradation of IкB $\alpha$ protein, as well as of the phosphorylation of NF- $\mathrm{\kappa B}$ p 65 and nuclear translocation of the activated NF- $\kappa \mathrm{B}$ p 65 subunit in cytokine-stimulated human keratinocytes. In addition, rf3 and rf4 effectively inhibited transcriptional activity of NF- $\kappa B$ p65. The differential inhibitory ability of rf3 and rf4 on the phosphorylation and degradation of $I \kappa B \alpha$ protein indicates that fraction $\mathrm{rf} 4$ may be targeting the upstream signaling cascade of I $\kappa \mathrm{B} / \mathrm{NF}-\kappa \mathrm{B}$ complex, including IKK complex or has dual inhibitory activities such as upstream cascade of IкB/NF- $\kappa \mathrm{B}$ complex and nuclear translocation of NF- $\kappa \mathrm{B}$. However, rf3 may target phosphorylation and/or nuclear translocation of NF- $\mathrm{KB}$ complex.

Inhibition of JAK/STAT, NF- $\mathrm{KB}$ and PI3K/Akt signaling by rf3 and rf4 resulted in the inhibition of the expression and production of pro-inflammatory mediators, including enzymes, adhesion molecules, cytokines and chemokines. Specifically, rf3 and rf4 strongly inhibited differentiation of Th2 and Th17 cell subsets by inhibition of the expression of Th2- and Th17-cell-lineage-specific master regulatory factors and their signature cytokines. These results may suggest potential therapeutic role of rf3 and rf4 for allergic and autoimmune diseases. ${ }^{15,16,21-23}$ To confirm these activities of rf3 and rf4, we examined the effects of these fractions in vivo on the psoriasis-like chronic inflammatory disease in mouse ears induced by intradermal injection of IL-23. Our results showed that topical application of rf3 and rf4 
effectively suppressed ear thickness and disease progression in this model.

In this study, rf3 and rf4, isolated from the aqueous methanol layer of Astragalus sinicus L., exhibited antioxidant and anti-inflammatory activities and regulation of immune responses in human keratinocytes and mouse $\mathrm{CD} 4{ }^{+} \mathrm{T}$ cells. The rf3 and rf4 inhibited JAK/STAT, NF- $\kappa \mathrm{B}$ and PI3K/Akt signaling. In addition, these fractions effectively inhibited differentiation of mouse CD4 ${ }^{+} \mathrm{T}$ cells into Th2 and Th17 cell subsets. Furthermore, we identified a therapeutic potential of rf3 and rf4 in cutaneous inflammatory disease induced by the IL-23-induced psoriasis model in mouse ears. Although we did not isolate and purify active single compounds from the fractions, we demonstrated the anti-inflammatory activity of Astragalus sinicus L. in experiments both in vitro and in vivo through regulation of the immune responses, and the results are the first report that Astragalus sinicus L. exhibits pharmacological activity.

\section{CONFLICT OF INTEREST}

The authors declare no conflict of interest.

\section{ACKNOWLEDGEMENTS}

This study was supported by a grant from the BioGreen 21 Program (PJ007175) of the Rural Development Administration, Republic of Korea. The funders had no role in study design, data collection and analysis, decision to publish or preparation of the manuscript.

1 O'Shea JJ, Park H, Pesu M, Borie D, Changelian P. New strategies for immunosuppression: interfering with cytokines by targeting the Jak/Stat pathway. Curr Opin Rheumatol 2005; 17: 305-311.

2 Terrell AM, Crisostomo PR, Wairiuko GM, Wang M, Morrell ED, Meldrum DR. Jak/STAT/SOCS signaling circuits and associated cytokine-mediated inflammation and hypertrophy in the heart. Shock 2006; 26: 226-234.

3 Cubbon RM, Ali N, Sengupta A, Kearney MT. Insulin- and growth factor-resistance impairs vascular regeneration in diabetes mellitus. Curr Vasc Pharmacol 2012; 10: 271-284.

4 Zhou A, Scoggin S, Gaynor RB, Williams NS. Identification of NF-kappa B-regulated genes induced by TNFalpha utilizing expression profiling and RNA interference. Oncogene 2003; 22: 2054-2064.

5 Oeckinghaus A, Hayden MS, Ghosh S. Crosstalk in NF-kB signaling pathways. Nat Immunol 2011; 12: 695-708.

6 Shuai K, Liu B. Regulation of JAK-STAT signalling in the immune system. Nat Rev Immunol 2003; 3: 900-911.
7 Karin M, Greten FR. NF-kappaB: linking inflammation and immunity to cancer development and progression. Nat Rev Immunol 2005; 5: 749-759.

8 Ito K, Caramori G, Adcock IM. Therapeutic potential of phosphatidylinositol 3-kinase inhibitors in inflammatory respiratory disease. J Pharmacol Exp Ther 2007; 321: 1-8.

9 Cui B, Inoue J, Takeshita T, Kinjo J, Nohara T. Triterpene glycosides from the seeds of Astragalus sinicus L. Chem Pharm Bull 1992; 40: 3330-3333.

10 Nagasawa H, Watanabe K, Yoshida M, Inatomi H. Effects of gold banded lily (Lilium auratum Lindl) or Chinese milk vetch (Astragalus sinicus $L$ ) on spontaneous mammary tumourigenesis in SHN mice. Anticancer Res 2001; 21: 2323-2328.

11 Kim Y, Kim BH, Lee H, Jeon B, Lee YS, Kwon MJ et al. Regulation of skin inflammation and angiogenesis by EC-SOD via HIF- $1 \alpha$ and NF-KB pathways. Free Radic Biol Med 2011; 51: 1985-1995.

$12 \mathrm{Kim} \mathrm{BH}, \mathrm{Na} \mathrm{KM}$, Oh I, Song IH, Lee YS, Shin J et al. Kurarinone regulates immune responses through regulation of the JAK/STAT and TCR-mediated signaling pathways. Biochem Pharmacol 2013; 85: $1134-1144$.

13 Kim BH, Lee JM, Jung YG, Kim S, Kim TY. Phytosphingosine Derivatives Ameliorate Skin Inflammation by Inhibiting NF-KB and JAK/STAT signaling in keratinocytes and mice. J Invest Dermatol (e-pub ahead of print 31 October 2013; doi:10.1038/jid.2013.453).

14 Lee YS, Cheon IS, Kim BH, Kwon MJ, Lee HW, Kim TY. Loss of extracellular superoxide dismutase induces severe IL-23-mediated skin inflammation in mice. J Invest Dermatol 2013; 133: 732-741.

15 Zhu J, Paul WE. Peripheral CD4 + T-cell differentiation regulated by networks of cytokines and transcription factors. Immunol Rev 2010; 238: 247-262.

16 Zhu J, Yamane H, Paul WE. Differentiation of effector CD4 T cell populations. Annu Rev Immunol 2010; 28: 445-489.

17 Zhu J, Paul WE. CD4 T cells: fates, functions, and faults. Blood 2008; 112: $1557-1569$.

18 Tak PP, Firestein GS. NF-kappaB: a key role in inflammatory diseases. J Clin Invest 2001; 107: 7-11.

19 Hedrick MN, Lonsdorf AS, Shirakawa AK, Richard Lee CC, Liao F, Singh SP et al. CCR6 is required for IL-23-induced psoriasis-like inflammation in mice. J Clin Invest 2009; 119: 2317-2329.

20 Rizzo HL, Kagami S, Phillips KG, Kurtz SE, Jacques SL, Blauvelt A. IL-23mediated psoriasis-like epidermal hyperplasia is dependent on IL-17A. J Immunol 2011; 186: 1495-1502.

21 Abbas AK, Murphy KM, Sher A. Functional diversity of helper T lymphocytes. Nature 1996; 383: 787-793.

22 Dong C. Diversification of T-helper-cell lineages: finding the family root of IL-17-producing cells. Nat Rev Immunol 2006; 6: 329-333.

23 Iwakura Y, Ishigame H. The IL-23/IL-17 axis in inflammation. J Clin Invest 2006; 116: 1218-1222.

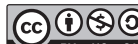
This work is licensed under a Creative Commons Attribution-NonCommercial-ShareAlike 3.0 Un- ported License. To view a copy of this license, visit http:// creativecommons.org/licenses/by-nc-sa/3.0/

Supplementary Information accompanies the paper on Experimental \& Molecular Medicine website (http://www.nature.com/emm) 